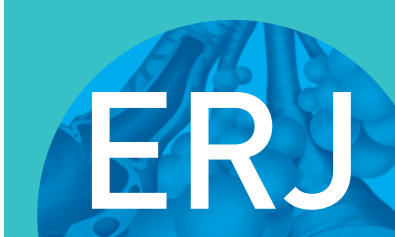

open research

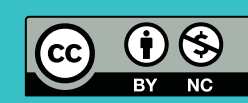

\section{The impact of muscle strength on exercise capacity and symptoms}

\author{
Mohammed Abdul Malik Farooqi ${ }^{1,2}{ }^{1,}$ Kieran Killian ${ }^{1}$ and Imran Satia ${ }^{1,2,3}$
}

Affiliations: ${ }^{1}$ McMaster University, Dept of Medicine, Division of Respirology, Hamilton, ON, Canada. ${ }^{2}$ Firestone Institute of Respiratory Health, St Joseph's Healthcare, Hamilton, ON, Canada. ${ }^{3}$ University of Manchester, Division of Infection, Immunity and Respiratory Medicine, and Manchester Academic Health Science Centre, Manchester, UK.

Correspondence: M.A. Malik Farooqi, McMaster University Medical Centre, 3U 21-26, 1280 Main St West, Hamilton, ON, L8S 4LA, Canada. E-mail: malik.farooqiamedportal.ca

ABSTRACT The capacity to exercise is a major contributor to functional limitation and is accompanied by increased morbidity and mortality. What are the most important physiological contributors to exercise capacity?

Cross-sectional data from consecutive patients referred to the McMaster University Medical Centre exercise laboratory for incremental cardiopulmonary exercise testing from 1988 to 2012 were analysed. Exercise capacity was determined by maximal power output (MPO) in $\mathrm{kpm} \cdot \mathrm{min}^{-1}$. The contributions of quadriceps strength (maximal peak force in $\mathrm{kg}$ using maximal dynamic voluntary contractions against hydraulic resistance), inspiratory muscle strength (determined using maximal inspiratory pressure (MIP)), maximal breathing capacity $(\mathrm{MBC})$ and gas exchange (carbon monoxide transfer coefficient $\left(K_{\mathrm{CO}}\right)$ ) were determined using regression coefficients in a multiple linear regression model. Dyspnoea and leg fatigue were measured using the modified Borg scale. Contributors to dyspnoea and leg fatigue were assessed using nonlinear regression.

A total of 36389 patients were included $(60 \%$ male, mean \pm sD age $53 \pm 18$ years). Mean \pm sD MPO, quadriceps strength and MIP achieved were $792 \pm 333 \mathrm{kpm} \cdot \mathrm{min}^{-1}, 46 \pm 18 \mathrm{~kg}$ and $75 \pm 31 \mathrm{cmH}_{2} \mathrm{O}$, respectively. MIP and quadriceps strength accounted for over half the variation in $M P O\left(R^{2}=0.57\right)$. Quadriceps strength was a stronger predictor of MPO (standardised regression coefficient, $\beta \pm \mathrm{SE}$ $0.37 \pm 0.005)$ than $\mathrm{MBC}(\beta \pm \mathrm{SE} 0.16 \pm 0.005)$ and $K_{\mathrm{CO}}(\beta \pm \mathrm{SE} 0.16 \pm 0.004)$, when adjusted for age, sex, height and weight. The effort required to cycle and breathe at any given power intensified systematically as both respiratory and peripheral muscle strength declined.

Muscle weakness causes exercise intolerance and should be routinely assessed in patients presenting with fatigue and dyspnoea, and those with functional limitation both in the presence or absence of disease.

@ERSpublications

Muscle strength is an important predictor of exercise capacity. It should be routinely assessed in patients presenting with fatigue and dyspnoea, and is an important domain of physical frailty. https://bit.ly/392i8Cn

Cite this article as: Farooqi MAM, Killian K, Satia I. The impact of muscle strength on exercise capacity and symptoms. ERJ Open Res 2020; 6: 00089-2020 [https://doi.org/10.1183/23120541.000892020].

Received: 24 Feb 2020 | Accepted after revision: 7 July 2020

Copyright $\odot$ ERS 2020. This article is open access and distributed under the terms of the Creative Commons Attribution Non-Commercial Licence 4.0. 


\section{Introduction}

The adverse health consequences of exercise intolerance are well described, and include increased morbidity from cardiovascular disease, diabetes and osteoporosis, increased healthcare utilisation, and increased mortality [1-4]. With an ageing population, the prevalence of exercise intolerance together with its contribution to functional limitation is expected to rise. Despite the presence of over 67 frailty instruments, no consensus criterion or assessment tool for frailty currently exists [5]. Frailty in the absence of weakness is difficult to imagine.

The physical frailty derived by Fried's criteria [6-8] is based on the assessment of weight loss, muscle weakness, exhaustion, slowness and reduced physical activity. Three of these domains (exhaustion, slowness and reduced physical activity) are closely associated with the capacity to exercise, and are typically determined by self-report and subject to bias. Muscle strength can be objectively and easily measured and is shown to be independently associated with adverse health outcomes including the development of cardiovascular disease and mortality [9]. Impairment in muscle strength may be a fundamental cause of functional limitation. The rate of fatigue for all muscles intensifies whenever the physiological support systems required decline or fail (i.e. cardiovascular, ventilatory, gas exchange, biochemical and metabolic). Independently of this support, all muscles fatigue more rapidly as their strength decreases. The contribution of muscle strength compared to these physiological systems in patients presenting with exercise limitation, fatigue and dyspnoea is often underappreciated. Muscle weakness is not commonly measured in clinical practice, nor is it currently recommended in guidelines on exercise testing when evaluating patients with dyspnoea, fatigue and reduced exercise capacity [10].

The objectives of this study were: 1) to investigate the contribution of quadriceps and inspiratory muscle strength to exercise limitation compared to ventilatory and gas exchange parameters and 2) determine the contributors to perceived effort required to cycle (fatigue) and breathe (dyspnoea) at incremental power outputs in a large cohort of both healthy subjects and patients with cardiac and respiratory disorders referred to a tertiary care exercise laboratory.

\section{Methods}

Study design and participants

Cross-sectional data from consecutive patients referred to the McMaster University Medical Centre exercise laboratory for incremental cardiopulmonary exercise testing from 1988 to 2012 were analysed. Baseline muscle strength and the physiological support parameters were measured during stepwise incremental exercise testing to capacity. All patients and healthy subjects with complete data for quadriceps and respiratory muscle strength and achieving a maximal power output (MPO) of $>100 \mathrm{kpm} \cdot \mathrm{min}^{-1}$ were included. Standard exercise testing was performed on patients according to American Thoracic Society guidelines [10]. The procedure and risks were explained to all patients and written informed consent obtained to exercise.

\section{Procedures}

Incremental exercise testing was performed on an electrically braked cycle ergometer with ECG monitoring (Siemens Elema 370; Siemens, Solna, Sweden) under the supervision of a trained pulmonary function and exercise technologists and cardiac technologists with the immediate availability of a physician. An incremental protocol to symptom limited capacity was used with $100 \mathrm{kpm} \cdot \mathrm{min}^{-1}$ increments. Subjects were strongly encouraged to exercise to exhaustion. MPO was defined as the highest power output in $\mathrm{kpm} \cdot \mathrm{min}^{-1}$ maintained for at least $30 \mathrm{~s}$. Subjects were asked to rate the intensity of the effort required to breathe (dyspnoea), the intensity of leg effort required to cycle (fatigue), and the intensity of chest pain or any other perceived discomfort by matching the intensity to semantic phrases tagged to numbers according to the modified Borg scale [11]. Spirometry included vital capacity (VC) and the maximum forced expiratory volume in $1 \mathrm{~s}\left(\mathrm{FEV}_{1}\right)$; maximum forced expired and inspired flow rates at $75 \%, 50 \%$ and $25 \%$ of the vital capacity. Maximal breathing capacity (MBC) was measured using the maximum tidal volume seen during exercise divided by the minimal time required to expire based on peak expiratory flow rate, forced expiratory flow at $50 \%$ lung volume $\left(\mathrm{FEF}_{50 \%}\right)$ and peak inspiratory flow rate [12]. Carbon monoxide transfer coefficient $\left(K_{\mathrm{CO}}\right)$ was measured using the standard single-breath method. Respiratory muscle strength was determined using maximal inspiratory pressure (MIP) measured at residual volume, and maximal expiratory pressure (MEP) measured at total lung capacity. Trials were accepted if repeat values were within 5\% of each other, with the larger two values used for analysis. Peripheral muscle strength was measured for the quadriceps, hamstrings, bench press and seated row using maximal dynamic voluntary contractions against hydraulic resistance (Hydrafitness Industries, Belton, TX, USA). The maximal peak force $(\mathrm{kg})$ was measured at the highest resistance and was quasi isokinetic due to a very positively accelerating force relative to the rate of movement ("Starling Resistor"). The quadriceps and MIP were the measures of muscle strength used for this study. 


\section{Statistical analysis}

Distribution of variables of interest MIP, quadriceps strength and MPO were evaluated by histogram and expressed as mean, SD and $\pm 95 \%$ confidence intervals. Statistical analysis was performed using univariate linear regression, multiple linear regression and nonlinear regression where appropriate. MPO, fatigue and dyspnoea were the outcomes of interest. Independent variables included peripheral and respiratory muscle strength parameters (quadriceps strength, MIP), baseline characteristics, ventilatory and gas exchange parameters. In order to illustrate the effects of muscle strength on MPO with greater clarity, we used multivariate ANOVA. The categories for quadriceps strength were $<20 \mathrm{~kg}, 20-40 \mathrm{~kg}, 40-60 \mathrm{~kg}, 60-80 \mathrm{~kg}$ and $>80 \mathrm{~kg}$ and for MIP were $<30,30-60,60-90$ and $>90 \mathrm{cmH}_{2} \mathrm{O}$. All analyses were completed using Statistica Academic (version 13).

\section{Results}

\section{Demographic characteristics}

In total, 36389 patients $(60 \%$ male, mean \pm SD age $53 \pm 18$ years) were included in the study. Descriptive statistics of the population are shown in table 1 . The distribution of quadriceps strength, inspiratory muscle strength and MPO followed a normal distribution in this population (figure 1). In the presence of both health and disease, the strength of the quadriceps, inspiratory and expiratory muscles and exercise capacity vary widely.

\section{Muscle strength and maximal power output}

Quadriceps strength and MIP alone accounted for 57\% of the variation in MPO (table 2). Covariates were sequentially added to this model, starting with baseline characteristics (age, sex, weight and height) followed by ventilation ( $\mathrm{MBC}$ and $\left.V_{\mathrm{A}}\right)$ and gas exchange $\left(K_{\mathrm{CO}}\right)$. Quadriceps muscle strength remained the strongest predictor of MPO after adjusting for these covariates (standardised regression coefficient, $\beta \pm \mathrm{SE}$ for quadriceps strength: $0.37 \pm 0.005$; MBC: $0.16 \pm 0.005$; and $\left.K_{\mathrm{CO}}: 0.16 \pm 0.004\right)$. The contribution of quadriceps and inspiratory muscle strength to MPO is shown in figure 2. This figure describes the multivariate ANOVA results and shows that incremental increases in both quadriceps strength and inspiratory muscle strength resulted in respective increases in power output. This suggests that both inspiratory muscle strength and quadriceps strength have a positive, linear relationship with power output.

The strength of one muscle group predicts the strength of all within a population, as seen in the correlation matrix that highlights the individual contribution of each factor to the MPO achieved and the interrelationship between the independent contributors (table 3). Quadriceps strength had the strongest correlation with MPO (Pearson $r=0.74$ ) and MIP correlated with MBC (Pearson $r=0.60$ ).

\section{TABLE 1 Baseline characteristics}

\begin{tabular}{|c|c|c|c|c|}
\hline & Mean & SD & 5th percentile & 95th percentile \\
\hline Height m & 1.69 & 0.10 & 1.52 & 1.85 \\
\hline Age years & 53.11 & 17.54 & 16.00 & 77.00 \\
\hline Weight kg & 78.58 & 18.73 & 50.80 & 110.20 \\
\hline BMI $\mathrm{kg} \cdot \mathrm{m}^{-2}$ & 27.41 & 5.44 & 19.45 & 37.13 \\
\hline MIP $\mathrm{cmH}_{2} \mathrm{O}$ & 74.66 & 30.54 & 30.00 & 130.00 \\
\hline MEP $\mathrm{cmH}_{2} \mathrm{O}$ & 106.36 & 0.70 & 0.70 & 2.97 \\
\hline Quads kg & 46.06 & 18.19 & 18.00 & 76.00 \\
\hline FEV $_{1}$ L BTPS & 2.74 & 0.88 & 1.40 & 4.30 \\
\hline VC L BTPS & 3.43 & 1.03 & 1.90 & 5.20 \\
\hline$D_{\text {Lco }} \mathrm{mL} \cdot \mathrm{mmHg}^{-1} \cdot \mathrm{min}^{-1}$ & 22.30 & 6.70 & 12.10 & 34.00 \\
\hline$K_{\text {co }} \mathrm{mL} \cdot \mathrm{mmHg}^{-1} \cdot \mathrm{min}^{-1} \cdot \mathrm{L}^{-1}$ & 4.39 & 0.99 & 2.84 & 6.06 \\
\hline$V_{\mathrm{A}}$ L BTPS & 5.15 & 1.33 & 3.00 & 7.30 \\
\hline MPO $\mathrm{kpm} \cdot \mathrm{min}^{-1}$ & 792.14 & 333.14 & 300.00 & 1400.00 \\
\hline
\end{tabular}

Total population $\mathrm{n}=36389,60 \%$ male. $\mathrm{BMI}$ : body mass index; MIP: maximal inspiratory pressure; MEP: maximal expiratory pressure; Quads: quadriceps strength; $\mathrm{FEV}_{1}$ : forced expiratory volume in $1 \mathrm{~s}$; BTPS: body temperature, ambient pressure, saturated with water vapour; VC: vital capacity; $D_{\mathrm{LCO}}$ : transfer capacity for carbon monoxide; $K_{\mathrm{CO}}$ : carbon monoxide transfer coefficient; $V_{\mathrm{A}}$ : alveolar volume; MPO: maximal power output. 

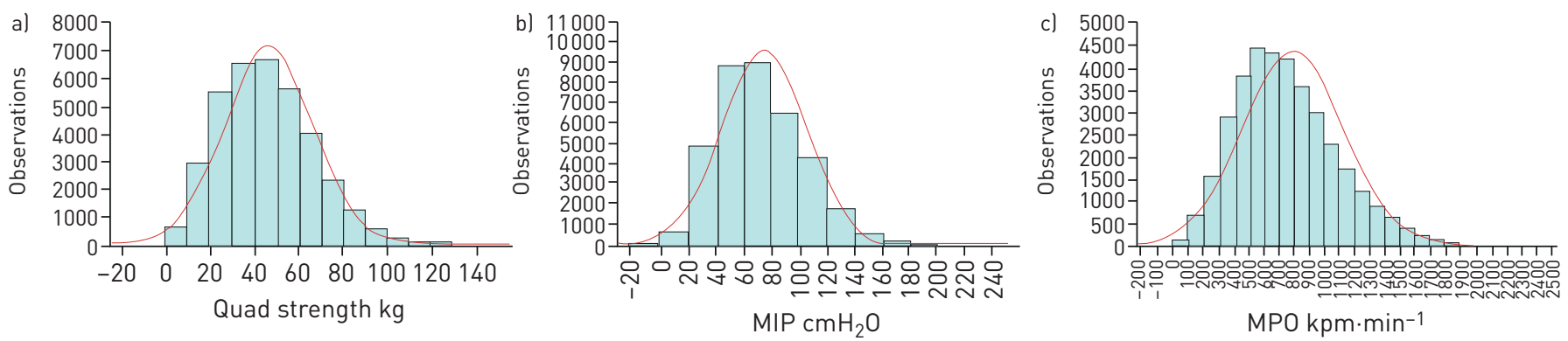

FIGURE 1 Distribution of a) quadriceps (Quad) strength, b) maximal inspiratory pressure (MIP) and c) maximal power output (MPO).

\section{Muscle strength and symptoms}

The perceived effort required to cycle and breathe at power outputs required during every day activity intensified systematically as both respiratory and peripheral muscle strength declined (figure 3). This shows that for power outputs required during daily activity, muscle strength is a key determinant of symptoms of dyspnoea and fatigue. This was modelled as: leg effort $=0.017 \times$ power $^{1.16} \times$ quads $^{-0.56}, \mathrm{r}=0.73$; breath effort $=0.016 \times$ power $^{1.06} \times \mathrm{MIP}^{-0.42}, \mathrm{r}=0.64$.

\section{Discussion}

This study demonstrates that leg and respiratory muscle strength are major contributors to the exercise capacity and symptom limitation during exercise in both health and disease. The novelty of this study is that we have taken an unbiased approach in including all subjects referred for cardiopulmonary exercise testing in a real-world setting irrespective of any underlying cardiac, respiratory or neuromuscular disorder. Our data promote the concept that exercise limitation is a consequence largely of deteriorating muscle strength; hence, assessment of muscle strength should strongly be considered when evaluating patients with poor functional status, impaired exercise tolerance and symptom limitation with exercise.

\section{Muscle strength, functional limitation and frailty}

Functional limitation is associated with adverse health outcomes and is often incorporated in models assessing and defining frailty, broadly conceptualised as a state of increased vulnerability to physiological stressors [13]. Traditional disease-specific risk factors such as those used in cardiovascular disease do not completely account for adverse outcomes [14, 15], and the expected epidemiological transitions that result from an ageing population suggest that a frail state independently accounts for these outcomes [16, 17]

TABLE 2 Multivariate regression analysis using maximal power output (MPO) as the dependent variable and muscle strength measures (quadriceps and maximal inspiratory pressure (MIP)) as independent variables

\begin{tabular}{|c|c|c|c|c|c|c|}
\hline \multirow[t]{2}{*}{ Dependent variable MPO (kpm $\left.\cdot \mathrm{min}^{-1}\right)$} & \multicolumn{2}{|c|}{$\begin{array}{c}\text { Unadjusted } \\
R=0.76, R^{2}=0.57\end{array}$} & \multicolumn{2}{|c|}{$\begin{array}{l}\text { Adjusted for baseline } \\
\text { characteristics } \\
R=0.80, R^{2}=0.63\end{array}$} & \multicolumn{2}{|c|}{$\begin{array}{c}\text { Adjusted for baseline } \\
\text { characteristics and ventilation } \\
R=0.83, R^{2}=0.68\end{array}$} \\
\hline & B (SE) & $\beta$ (SE) & $B$ (SE) & $\beta$ (SE) & B (SE) & $\beta$ (sE) \\
\hline Intercept & $161.5(3.2)$ & 0 & $-874.7(22.2)$ & 0 & $-721.3(28.5)$ & 0 \\
\hline Quadriceps strength kg & $10.0(0.1)$ & $0.61(0.004)$ & 7.68 (0.08) & $0.47(0.005)$ & $6.16(0.09)$ & $0.37(0.005)$ \\
\hline $\mathrm{MIP} \mathrm{cmH}_{2} \mathrm{O}$ & $2.25(0.05)$ & $0.21(0.004)$ & $1.77(0.04)$ & $0.16(0.005)$ & $1.01(0.05)$ & $0.09(0.005)$ \\
\hline Height $\mathrm{m}$ & & & $785.4(14.9)$ & $0.24(0.005)$ & $342(18.8)$ & $0.10(0.006)$ \\
\hline Weight kg & & & $-0.81(0.07)$ & $-0.05(0.004)$ & $-1.13(0.07)$ & $-0.06(0.004)$ \\
\hline Sex female & & & & & $-9.96(3.21)$ & $-0.001(0.005)$ \\
\hline MBC L & & & & & $1.10(0.04)$ & $0.16(0.005)$ \\
\hline$V_{\text {A }}$ L BTPS & & & & & $59.8(1.47)$ & $0.27(0.005)$ \\
\hline$K_{\mathrm{co}} \mathrm{mL} \cdot \mathrm{mmHg}^{-1} \cdot \mathrm{min}^{-1} \cdot \mathrm{L}^{-1}$ & & & & & $56.4(1.35)$ & $0.16(0.004)$ \\
\hline
\end{tabular}


FIGURE 2 Effect of variations in quadriceps strength (quads) and maximal inspiratory pressure (MIP) on maximal power output (MPO) during incremental cardiopulmonary exercise testing. Results obtained from multivariate ANOVA analysis using MPO as the independent variable and MIP and quads as categorical dependent variables. Data shown as mean and $95 \% \mathrm{Cl}$.

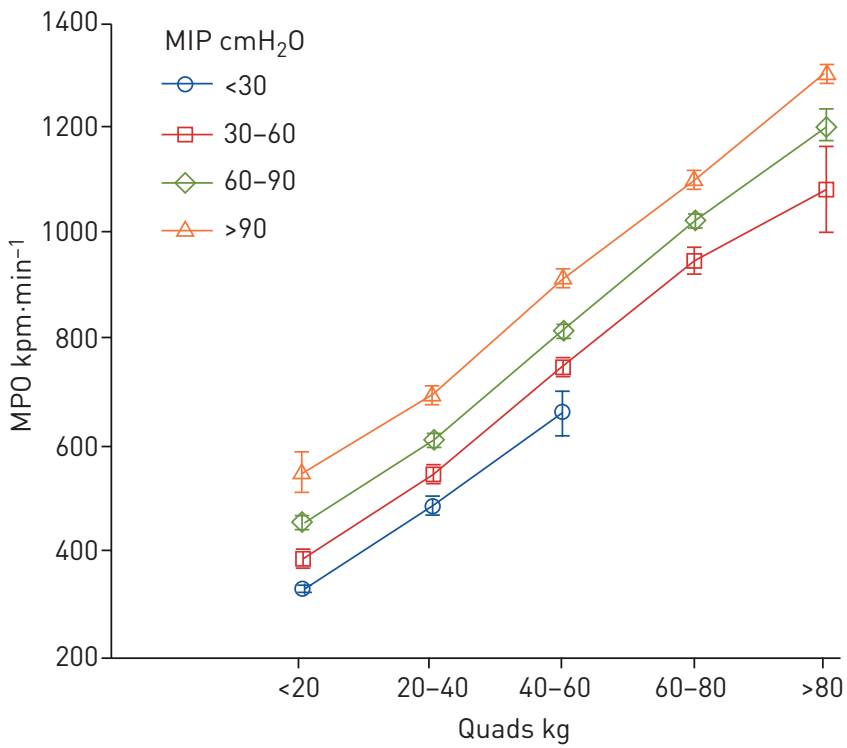

Therefore, it is critical to develop clinically useful tools to measure frailty to allow clinicians and researchers to optimise risk estimation and healthcare delivery. This is supported in white paper statements from several medical societies [18-20]. Despite this, there continues to be a lack of consensus around the best method to operationalise frailty, and most tools incorporate subjective, psychometric assessments of self-reported physical activity and exhaustion that are susceptible to responder bias [18, 21]. Physiological evaluation of frailty instruments and their components are needed. Our study assesses the physiological contribution of muscle strength, a key criterion used in the physical frailty phenotype model [6].

Muscle strength can be simply and objectively measured using tools such as the dynamometer that have been well standardised with age- and sex-based reference values [22]. As such, some authors have recommended muscle strength assessment alone as a standardised way of screening for physical frailty and as a reliable outcome measure for clinical trials [23, 24]. Muscle weakness is a consequence of deconditioning and a downstream effect of comorbid disease. This is supported by direct, independent associations of muscle strength with adverse health outcomes, including mortality [25]; however, few studies have described its physiological contribution to exercise limitation and symptoms at day-to-day levels of power output. One study in 33 patients with heart failure ( $81 \%$ men, mean age $60 \pm 12$ years) described quadriceps strength as the main physiological parameter correlating with power output and symptom limitation [26]. Similar studies that evaluated the contribution of muscle strength using cardiopulmonary exercise testing (CPET) as the measure of exercise capacity have been conducted in patients with COPD have been small in size $(n<100)$ [27]. No study to date has evaluated a comprehensive, real-world cohort of both healthy patients and those with cardiorespiratory conditions.

TABLE 3 Univariate correlation matrix of maximal power output (MPO), muscle strength and lung diffusion and maximal breathing capacity (MBC)

$\begin{array}{lccccccccc}\text { Variable } & \text { MPO } & \text { Row } & \text { Bench } & \text { Quads } & \text { MIP } & \text { MEP } & \boldsymbol{K}_{\text {co }} & \boldsymbol{V}_{\text {A }} & \text { MBC } \\ \text { MPO } & 1.00 & 0.66 & 0.74 & 0.74 & 0.58 & 0.53 & 0.22 & 0.60 & 0.67 \\ \text { Row } & 0.66 & 1.00 & 0.86 & 0.80 & 0.59 & 0.63 & 0.05 & 0.63 & 0.63 \\ \text { Bench } & 0.74 & 0.86 & 1.00 & 0.84 & 0.63 & 0.64 & 0.10 & 0.64 & 0.67 \\ \text { Quads } & 0.74 & 0.80 & 0.84 & 1.00 & 0.61 & 0.61 & 0.11 & 0.60 & 0.65 \\ \text { MIP } & 0.58 & 0.59 & 0.63 & 0.61 & 1.00 & 0.69 & 0.11 & 0.45 & 0.60 \\ \text { MEP } & 0.53 & 0.63 & 0.64 & 0.61 & 0.69 & 1.00 & 0.01 & 0.46 & 0.53 \\ \text { K } & 0.22 & 0.05 & 0.10 & 0.11 & 0.11 & 0.01 & 1.00 & -0.24 & 0.03 \\ \text { V }_{\text {M }} & 0.60 & 0.63 & 0.64 & 0.60 & 0.45 & 0.46 & -0.24 & 1.00 & 0.70 \\ \text { MBC } & 0.67 & 0.63 & 0.67 & 0.65 & 0.60 & 0.53 & 0.03 & 0.70 & 1.00\end{array}$

Data shown as Pearson $r$ correlations. Quads: quadriceps strength; MIP: maximal inspiratory pressure; MEP: maximal expiratory pressure; $K_{\mathrm{co}}$ : carbon monoxide transfer coefficient; $V_{\mathrm{A}}$ : alveolar volume. 

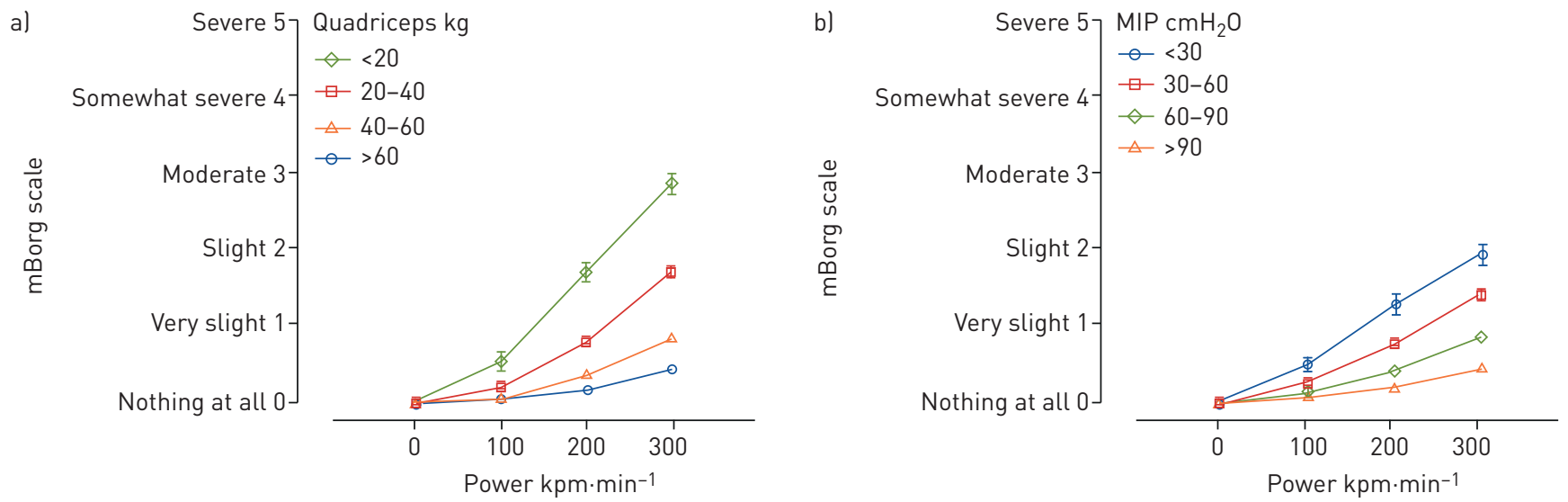

FIGURE 3 Subjective discomfort in a) leg effort and b) dyspnoea during exercise, stratified by muscle strength (quadriceps and maximal inspiratory pressure (MIP)). Results selected for power outputs required during daily activities. Discomfort measured on a modified Borg scale. Data shown as mean and $95 \% \mathrm{Cl}$.

Such paucity of data may stem from guideline statements failing to emphasise the measurement of muscle strength as part of routine CPET [10], despite early studies recommending its use [28, 29]. The conventional approach to CPET assumes fatigue and evolving weakness as a consequence of cardiopulmonary and other impairments and baseline strength goes underappreciated.

\section{Effort, symptoms and weakness}

The conscious sense of effort is generated by the intensity of the central motor command and not by afferent information relating to the tension, displacement or metabolic status of the muscle [30]. Common experience shows that a leg paralysed by a stroke leading to failure of transmission of the centrally generated motor command is reported by patients as heavy (i.e. due to the increased sense of effort required to move the leg). Likewise, the effort and the intensity of the central motor command required to generate power is much greater in those with weak muscles. Furthermore, maximum effort does not generate the same maximal power, which is dependent on the strength and amount of muscle available for activation. These variations in muscle strength across individuals are obvious but seldom used to explain exercise intolerance. Similarly, respiratory muscle weakness results in an increased motor command required to perform the increased ventilatory workload during exercise, and contributes to the multifactorial sensation of dyspnoea [31]. Our data support this view that subjects with weaker muscles become more breathless and develop more leg fatigue at equal work rates between individuals, irrespective of underlying cardiac and respiratory disease.

\section{Strengths and limitations}

Our study explores the relationship between peripheral and respiratory muscle strength, exercise capacity and symptom limitation (fatigue and dyspnoea) associated with exercise in a large, real-life cohort of patients evaluated with comprehensive CPET. We systematically describe the significant contribution of muscle strength to effort and functional limitation and explain the importance of these findings in the context of the increasingly recognised health burden of physical frailty. Despite these strengths, our study has a few limitations. First, this is a cross-sectional study performed in one centre. It is unclear whether similar results would be demonstrated prospectively within an individual over time. Secondly, underlying comorbidities were not described in detail; however, by focusing on physiological contributors, we present a much broader view of exercise limitation and its relationship with muscle strength. Third, we did not measure frailty using an operational definition. Frailty is a term that is receiving increasing attention. By its nature it is difficult to define. Failure to respond to stressors is such a broad generalisation that it is scarcely possible to establish a robust experimental design where it can be refuted. In this sense, it lacks scientific credibility. The cardinal sin of science is trying to be precise about something that is inherently vague. The physiological implications of muscle weakness (a component of the most widely cited frailty instrument) have been highlighted in this study. Fourth, we did not assess the effects of the mechanical disadvantage due to altered lung volumes (hyperinflation) and its effects on muscle weakness. The relationships between respiratory muscle weakness and dyspnoea are more complicated than that of leg weakness and fatigue. Factors such as dynamic hyperinflation and ventilatory inefficiency contribute to dyspnoea but measurements of these were beyond the scope of this paper. Nonetheless, our results clearly show respiratory muscle weakness contributes to dyspnoea in all scenarios independently of the mechanics of 
airflow limitation. Finally, our measure of muscle strength (peak quadriceps force using maximal dynamic voluntary contractions against hydraulic resistance) is not a routinely accessible test of muscle function. There is a strong interdependence of one muscle group with others. Information may be extrapolated from other measures of muscle strength, such as grip strength, which are more commonly used.

\section{Conclusion}

Despite the focus on identifying and treating cardiac and respiratory conditions, muscle weakness is an underappreciated contributor to functional limitation in health and disease. Our results emphasise the importance of muscle strength assessment in the evaluation of physical frailty. In addition to cardiovascular and respiratory assessment, muscle strength should be assessed in patients presenting with exercise limitation, fatigue and dyspnoea. Further studies that describe effect sizes and cost-effectiveness of interventions that target strength improvement in functionally limited patients should be considered.

Author contributions: M.A.M. Farooqi, I. Satia and K. Killian conceived and designed the study, performed statistical analysis and modelling, reviewed the manuscript and approved the final draft.

Conflict of interest: None declared.

Support statement: I. Satia is supported by the European Respiratory Society Marie Curie Respire 3 Fellowship award. Funding information for this article has been deposited with the Crossref Funder Registry.

\section{References}

1 Vermeiren S, Vella-Azzopardi R, Beckwee D, et al. Frailty and the prediction of negative health outcomes: a meta-analysis. J Am Med Dir Assoc 2016; 17: 1163.e1-1163.e17.

2 Veronese N, Cereda E, Stubbs B, et al. Risk of cardiovascular disease morbidity and mortality in frail and pre-frail older adults: results from a meta-analysis and exploratory meta-regression analysis. Ageing Res Rev 2017; 35: 63-73.

3 Kojima G. Frailty as a predictor of hospitalisation among community-dwelling older people: a systematic review and meta-analysis. J Epidemiol Community Health 2016; 70: 722-729.

4 Kojima G. Frailty as a predictor of fractures among community-dwelling older people: a systematic review and meta-analysis. Bone 2016; 90: 116-122.

5 Buta BJ, Walston JD, Godino JG, et al. Frailty assessment instruments: systematic characterization of the uses and contexts of highly-cited instruments. Ageing Res Rev 2016; 26: 53-61.

6 Theou O, Cann L, Blodgett J, et al. Modifications to the frailty phenotype criteria: systematic review of the current literature and investigation of 262 frailty phenotypes in the survey of health, ageing, and retirement in Europe. Ageing Res Rev 2015; 21: 78-94.

7 Choi J, Ahn A, Kim S, et al. Global prevalence of physical frailty by Fried's criteria in community-dwelling elderly with national population-based surveys. J Am Med Dir Assoc 2015; 16: 548-550.

8 Fried LP, Tangen CM, Walston J, et al. Frailty in older adults: evidence for a phenotype. J Gerontol A Biol Sci Med Sci 2001; 56: M146-M157.

9 Leong DP, Teo KK, Rangarajan S, et al. Prognostic value of grip strength: findings from the Prospective Urban Rural Epidemiology (PURE) study. Lancet 2015; 386: 266-273.

10 American Thoracic Society; American College of Chest Physicians. ATS/ACCP statement on cardiopulmonary exercise testing. Am J Respir Crit Care Med 2003; 167: 211-277.

11 Wilson RC, Jones PW. A comparison of the visual analogue scale and modified Borg scale for the measurement of dyspnoea during exercise. Clin Sci 1989; 76: 277-282.

12 Bartlett RG Jr, Specht H. Maximum breathing capacity with various expiratory and inspiratory resistances (single and combined) at various breathing rates. J Appl Physiol 1957; 11: 79-83.

13 Xue Q-L. The frailty syndrome: definition and natural history. Clin Geriatr Med 2011; 27: 1-15.

14 Yusuf S, Joseph P, Rangarajan S, et al. Modifiable risk factors, cardiovascular disease, and mortality in 155722 individuals from 21 high-income, middle-income, and low-income countries (PURE): a prospective cohort study. Lancet 2020; 395: 795-808.

15 Faroogi MAM, Gerstein H, Yusuf S, et al. Accumulation of deficits as a key risk factor for cardiovascular morbidity and mortality: a pooled analysis of 154000 individuals. J Am Heart Assoc 2020; 9: e014686.

16 Kojima G, Iliffe S, Walters K. Frailty index as a predictor of mortality: a systematic review and meta-analysis. Age Ageing 2018; 47: 193-200.

17 Vaes B, Depoortere D, Van Pottelbergh G, et al. Association between traditional cardiovascular risk factors and mortality in the oldest old: untangling the role of frailty. BMC Geriatr 2017; 17: 234.

18 Morley JE, Vellas B, Van Kan GA, et al. Frailty consensus: a call to action. J Am Med Dir Assoc 2013; 14 392-397.

19 Afilalo J, Alexander KP, Mack MJ, et al. Frailty assessment in the cardiovascular care of older adults. J Am Coll Cardiol 2014; 63: 747-762.

20 Singer JP, Lederer DJ, Baldwin MR. Frailty in pulmonary and critical care medicine. Ann Am Thorac Soc 2016; 13: 1394-1404.

21 Elasy TA, Gaddy G. Measuring subjective outcomes: rethinking reliability and validity. J Gen Intern Med 1998; 13 757-761.

22 Bohannon RW. Reference values for extremity muscle strength obtained by hand-held dynamometry from adults aged 20 to 79 years. Arch Phys Med Rehabil 1997; 78: 26-32.

23 Cruz-Jentoft AJ, Michel JP. Sarcopenia: a useful paradigm for physical frailty. Eur Geriatr Med 2013; 4: 102-105. 
24 Syddall H, Cooper C, Martin F, et al. Is grip strength a useful single marker of frailty? Age Ageing 2003; 32: 650-656.

25 Leong DP, Teo KK, Rangarajan S, et al. Prognostic value of grip strength: findings from the Prospective Urban Rural Epidemiology (PURE) study. Lancet 2015; 386: 266-273.

26 Suzuki K, Omiya K, Yamada S, et al. Relations between strength and endurance of leg skeletal muscle and cardiopulmonary exercise testing parameters in patients with chronic heart failure. J Cardiol 2004; 43: 59-68.

27 Gosselink R, Troosters T, Decramer M. Peripheral muscle weakness contributes to exercise limitation in COPD. Am J Respir Crit Care Med 1996; 153: 976-980.

28 Hamilton AL, Killian KJ, Summers E, et al. Muscle strength, symptom intensity, and exercise capacity in patients with cardiorespiratory disorders. Am J Respir Crit Care Med 1995; 152: 2021-2031.

29 Jones NL, Killian KJ. Exercise limitation in health and disease. N Engl J Med 2000; 343: 632-641.

30 de Morree HM, Klein C, Marcora SM. Perception of effort reflects central motor command during movement execution. Psychophysiology 2012; 49: 1242-1253.

31 Killian KJ, Campbell EJ. Dyspnea and exercise. Annu Rev Physiol 1983; 45: 465-479. 